$\underset{\text { publications }}{\mathbf{A}} \underset{\mathbf{C}}{\mathbf{C}} \mathbf{G}$

Rec. Nat. Prod. 13:5 (2019) 413-417 records of natural products

\title{
Structural Elucidation of a Coumarin with New Skeleton from Artemisia ordosica
}

\section{Wugedunqiqige Bao ${ }^{\circledR}$, Qinghu Wang * and Junsheng Hao}

\author{
College of Traditional Mongolian Medicine, Inner Mongolia University for Nationalities, \\ Tongliao, Inner Mongolia 028000, P. R. China
}

(Received October 31, 2018; Revised January 30, 2019; Accepted February 01, 2019)

\begin{abstract}
A new coumarin, named as arteordocoumarin A (1), together with eight known compounds (2-9) were isolated from the $\mathrm{CHCl}_{3}$ extract of Artemisia ordosica (A. ordosica). The structures of $\mathbf{1}$ was elucidated by spectroscopic methods, including UV, IR, HR-ESI-MS and extensive 1D and 2D NMR techniques.
\end{abstract}

Keywords: Arteordocoumarin A; Artemisia ordosica; NMR. (C) 2019 ACG Publications. All rights reserved.

\section{Introduction}

A. ordosica, Asteraceae family, is one of the main arido-active shrubs growing in the arid and semi-arid areas of the north China including Inner Mongolia, Ningxia, Gansu and Shanxi $[1,2]$. The aerial parts of A. ordosica is utilized as a folk medicine for expelling rheumatism, clearing heat, and dispelling swelling [3]. Sterols [4], coumarins [5], terpenoids [6], flavonoids [7, 8] and acetylenes [3] were isolated previously from this plant. However, the secondary metabolites from Artemisia ordosica often differ when grown in different ecological environments. In order to continue our research on the bioactive secondary metabolites from Artemisia ordosica collected in Tongliao of Inner Mongolia, China, we now describe the isolation and structure elucidation of a new coumarin compound, together with eight known ones.

\section{Materials and Methods}

\subsection{Instrumentation and Reagents}

A Shimadzu UV-2201 spectrometer (Shimadzu, Japan) was used to record the he UV spectra. The IR spectra were recorded in $\mathrm{KBr}$ discs on a Thermo Nicolet 200 double beam spectrophotometer (Shimadzu, Japan). A Waters Xevo G2-S QT (Waters, USA) was used to measure the HR-ESI-MS spectra. NMR spectra were measured on a Bruker AV-500 spectrometer (Bruker, Germany) with tetramethylsilane (TMS) as the internal reference, and chemical shifts are expressed in $\delta(\mathrm{ppm})$. Column

\footnotetext{
*Corresponding author: E-Mail: wqh196812@163.com; Phone:086-0475-8314242 Fax:086-0475-8314242
} 
chromatography was performed by using silica gel (200-300 mesh, Marine Chemical Factory, Qingdao, China).

\subsection{Plant Materials}

The aerial parts of A. ordosica were collected in Tongliao, Inner Mongolia of China, in June 2017, and identified by Prof. Buhebateer (Inner Mongolia University for Nationalities). A voucher (ref. no. 20170612) has been deposited at the Herbarium of college of Traditional Mongolian Medicine of Inner Mongolia University for Nationalities.

\subsection{Extraction}

Ground-dried aerial parts of A. ordosica $(2.0 \mathrm{~kg})$ were extracted with $\mathrm{CHCl}_{3}(25 \mathrm{~L})$ under reflux after extraction with $10 \mathrm{~L}$ petroleum ether. Evaporation of the solvent under reduced pressure delivered the $\mathrm{CHCl}_{3}$ extract $(150.0 \mathrm{~g})$. The $\mathrm{CHCl}_{3}$ extract was fractionated by column chromatography on silica gel and gradiently eluted with petroleum ether- $\mathrm{CH}_{3} \mathrm{COCH}_{3}$ (60:1 to 20:1) to give 3 fractions (Fr. 1-3). Fr. $1(320 \mathrm{mg}$ ) was separated by TLC (cyclohexane-ethyl acetate, 10:1) yielding $1(17 \mathrm{mg}), 2(11 \mathrm{mg})$ and $3(15 \mathrm{mg})$; Fr. $2(400.0 \mathrm{mg})$ was further eluted on a Sephadex LH-20 column with $\mathrm{MeOH}: \mathrm{CHCl}_{3}$ $(v: v, 1: 1)$ and then separated by TLC (cyclohexane-ethyl acetate, 7:1) yielding $4(13 \mathrm{mg}), \mathbf{5}(9 \mathrm{mg}), \mathbf{6}$ $(15 \mathrm{mg}$ ) and $7(18 \mathrm{mg})$. Fr. 3 (330 mg) was separated by TLC (cyclohexane-ethyl acetate, 3:1) yielding $\mathbf{8}(16 \mathrm{mg})$ and $9(21 \mathrm{mg})$.

Arteordocoumarin A (1): White needle; ${ }^{1} \mathrm{H}-\mathrm{NMR}\left(500 \mathrm{MHz}, \mathrm{DMSO}-\mathrm{d}_{6}\right)$ and ${ }^{13} \mathrm{C}-\mathrm{NMR}(125 \mathrm{MHz}$, DMSO-d ${ }_{6}$ ) spectral data see Table 1; HR-ESI-MS at $\mathrm{m} / z 209.0467$ [M-H] $]^{-}$(calcd for $\mathrm{C}_{10} \mathrm{H}_{9} \mathrm{O}_{5}$, 209.0450).

6,7-dimethoxycoumarin (2) [9]: White needle; ${ }^{1} \mathrm{H}-\mathrm{NMR}\left(500 \mathrm{MHz}, \mathrm{CDCl}_{3}\right) \delta_{\mathrm{H}}: 6.31(1 \mathrm{H}, \mathrm{d}, J=9.5 \mathrm{~Hz}$, $\mathrm{H}-3), 7.65(1 \mathrm{H}, \mathrm{d}, J=9.5 \mathrm{~Hz}, \mathrm{H}-4), 6.86(1 \mathrm{H}, \mathrm{s}, \mathrm{H}-5), 6.88(1 \mathrm{H}, \mathrm{s}, \mathrm{H}-8), 3.94\left(3 \mathrm{H}, \mathrm{s}, 6-\mathrm{OCH}_{3}\right), 3.97$ $\left(3 \mathrm{H}, \mathrm{s}, 7-\mathrm{OCH}_{3}\right) ;{ }^{13} \mathrm{C}-\mathrm{NMR}\left(125 \mathrm{MHz}, \mathrm{CDCl}_{3}\right) \delta_{\mathrm{C}}: 161.5(\mathrm{C}-2), 113.6(\mathrm{C}-3), 143.3(\mathrm{C}-4), 107.9(\mathrm{C}-5)$, 146.3 (C-6), 152.8 (C-7), 100.0 (C-8), 150.0 (C-9), $111.4(\mathrm{C}-10), 56.4\left(6-\mathrm{OCH}_{3}\right), 56.3\left(7-\mathrm{OCH}_{3}\right)$.

6,7,8-trimethoxycoumarin (3) [10]: White needle; ${ }^{1} \mathrm{H}-\mathrm{NMR}\left(500 \mathrm{MHz}, \mathrm{CDCl}_{3}\right) \delta_{\mathrm{H}}: 6.06(1 \mathrm{H}, \mathrm{d}, J=9.0$ $\mathrm{Hz}, \mathrm{H}-3), 7.52(1 \mathrm{H}, \mathrm{d}, J=9.0 \mathrm{~Hz}, \mathrm{H}-4), 7.30(1 \mathrm{H}, \mathrm{s}, \mathrm{H}-5), 3.88\left(3 \mathrm{H}, \mathrm{s}, 6-\mathrm{OCH}_{3}\right), 3.86\left(3 \mathrm{H}, \mathrm{s}, 8-\mathrm{OCH}_{3}\right)$, $3.82\left(3 \mathrm{H}, \mathrm{s}, 7-\mathrm{OCH}_{3}\right) ;{ }^{13} \mathrm{C}-\mathrm{NMR}\left(125 \mathrm{MHz}, \mathrm{CDCl}_{3}\right) \delta_{\mathrm{C}}: 165.4(\mathrm{C}-2), 115.5(\mathrm{C}-3), 140.3(\mathrm{C}-4), 114.9(\mathrm{C}-$ 5), 145.0 (C-6), 125.1 (C-7), 133.5 (C-8), 134.4 (C-9), 120.7 (C-10), $56.4\left(6-\mathrm{OCH}_{3}\right), 56.1\left(8-\mathrm{OCH}_{3}\right)$, $55.8\left(7-\mathrm{OCH}_{3}\right)$.

6-hydroxy-7-methoxycoumarin (4) [9]: White needle; ${ }^{1} \mathrm{H}-\mathrm{NMR}\left(500 \mathrm{MHz}, \mathrm{CDCl}_{3}\right) \delta_{\mathrm{H}}$ : $6.31(1 \mathrm{H}, \mathrm{d}, J=$ 9.5 Hz, H-3), $7.64(1 \mathrm{H}, \mathrm{d}, J=9.5 \mathrm{~Hz}, \mathrm{H}-4), 6.93(1 \mathrm{H}, \mathrm{s}, \mathrm{H}-5), 6.85(1 \mathrm{H}, \mathrm{s}, \mathrm{H}-8), 3.91\left(3 \mathrm{H}, \mathrm{s}, 7-\mathrm{OCH}_{3}\right)$; ${ }^{13} \mathrm{C}-\mathrm{NMR}\left(125 \mathrm{MHz}, \mathrm{CDCl}_{3}\right) \delta_{\mathrm{C}}: 161.6(\mathrm{C}-2), 113.5(\mathrm{C}-3), 143.5(\mathrm{C}-4), 108.3(\mathrm{C}-5), 147.3(\mathrm{C}-6), 152.0$ (C-7), 103.0 (C-8), 153.0 (C-9), $113.4(\mathrm{C}-10), 56.5\left(7-\mathrm{OCH}_{3}\right)$.

4-hydroxylacetophenone (5) [11]: White needle; ${ }^{1} \mathrm{H}-\mathrm{NMR}\left(500 \mathrm{MHz}, \mathrm{CDCl}_{3}\right) \delta_{\mathrm{H}}: 7.93(2 \mathrm{H}, \mathrm{d}, J=8.5$ $\mathrm{Hz}, \mathrm{H}-2,6), 6.91(2 \mathrm{H}, \mathrm{d}, J=8.5 \mathrm{~Hz}, \mathrm{H}-3,5), 2.58\left(3 \mathrm{H}, \mathrm{s},-\mathrm{CH}_{3}\right) ;{ }^{13} \mathrm{C}-\mathrm{NMR}\left(125 \mathrm{MHz}, \mathrm{CDCl}_{3}\right) \delta_{\mathrm{C}}: 130.4$ (C-1), 131.0 (C-2), 115.3 (C-3), $160.1(\mathrm{C}-4), 115.3(\mathrm{C}-5), 131.0(\mathrm{C}-6), 197.9(\mathrm{C}=\mathrm{O}), 26.3\left(-\mathrm{CH}_{3}\right)$.

4-hydroxy-5-methoxylacetophenone (6) [11]: White needle; ${ }^{1} \mathrm{H}-\mathrm{NMR}\left(500 \mathrm{MHz}, \mathrm{CDCl}_{3}\right) \delta_{\mathrm{H}}: 7.59(1 \mathrm{H}$, $\mathrm{d}, J=2.0 \mathrm{~Hz}, \mathrm{H}-2), 6.98(1 \mathrm{H}, \mathrm{d}, J=8.0 \mathrm{~Hz}, \mathrm{H}-5), 7.62(1 \mathrm{H}, \mathrm{d}, J=8.0,2.0 \mathrm{~Hz}, \mathrm{H}-6), 3.90(3 \mathrm{H}, \mathrm{s},-$ $\left.\mathrm{OCH}_{3}\right), 2.51\left(3 \mathrm{H}, \mathrm{s},-\mathrm{CH}_{3}\right) ;{ }^{13} \mathrm{C}-\mathrm{NMR}\left(125 \mathrm{MHz}, \mathrm{CDCl}_{3}\right) \delta_{\mathrm{C}}: 129.9(\mathrm{C}-1), 111.1(\mathrm{C}-2), 147.0(\mathrm{C}-3), 146.8$ (C-4), $114.4(\mathrm{C}-5), 126.9(\mathrm{C}-6), 190.3(\mathrm{C}=\mathrm{O}), 56.8\left(-\mathrm{OCH}_{3}\right), 26.3\left(-\mathrm{CH}_{3}\right)$.

4-hydroxybenzaldehyde (7) [12]: White needle; ${ }^{1} \mathrm{H}-\mathrm{NMR}\left(500 \mathrm{MHz}, \mathrm{CDCl}_{3}\right) \delta_{\mathrm{H}}: 7.82(2 \mathrm{H}, \mathrm{d}, J=8.5 \mathrm{~Hz}$, $\mathrm{H}-2,6), 7.02(2 \mathrm{H}, \mathrm{d}, J=8.5 \mathrm{~Hz}, \mathrm{H}-3,5), 9.85(1 \mathrm{H}, \mathrm{s},-\mathrm{CHO}) ;{ }^{13} \mathrm{C}-\mathrm{NMR}\left(125 \mathrm{MHz}, \mathrm{CDCl}_{3}\right) \delta_{\mathrm{C}}: 139.3(\mathrm{C}-$ 1), 132.5 (C-2), 116.0 (C-3), 162.0 (C-4), 116.0 (C-5), 132.5 (C-6), 191.3 (-CHO). 
4-hydroxy-5-methoxybenzaldehyde (8) [13]: White needle; ${ }^{1} \mathrm{H}-\mathrm{NMR}\left(500 \mathrm{MHz}, \mathrm{CDCl}_{3}\right) \delta_{\mathrm{H}}: 7.43(1 \mathrm{H}$, brs, H-2), $7.05(1 \mathrm{H}, \mathrm{d}, J=8.5 \mathrm{~Hz}, \mathrm{H}-5), 7.45(1 \mathrm{H}, \mathrm{brd}, J=8.5 \mathrm{~Hz}, \mathrm{H}-6), 9.83(1 \mathrm{H}, \mathrm{s},-\mathrm{CHO}), 3.99$ (3H, $\left.\mathrm{s},-\mathrm{OCH}_{3}\right) ;{ }^{13} \mathrm{C}-\mathrm{NMR}\left(125 \mathrm{MHz}, \mathrm{CDCl}_{3}\right) \delta_{\mathrm{C}}: 129.9(\mathrm{C}-1), 108.8(\mathrm{C}-2), 151.7(\mathrm{C}-3), 147.2(\mathrm{C}-4), 114.4$ (C-5), $127.6(\mathrm{C}-6), 191.0(\mathrm{C}=\mathrm{O}), 56.1\left(-\mathrm{OCH}_{3}\right)$.

4,5-dihydroxybenzaldehyde (9) [13]: ${ }^{1} \mathrm{H}-\mathrm{NMR}\left(500 \mathrm{MHz}, \mathrm{CDCl}_{3}\right) \delta_{\mathrm{H}}: 7.44(1 \mathrm{H}, \mathrm{d}, J=2.0 \mathrm{~Hz}, \mathrm{H}-2), 6.96$ $(1 \mathrm{H}, \mathrm{d}, J=8.0 \mathrm{~Hz}, \mathrm{H}-5), 7.46(1 \mathrm{H}, \mathrm{brd}, J=8.0,2.0 \mathrm{~Hz}, \mathrm{H}-6), 9.85(1 \mathrm{H}, \mathrm{s},-\mathrm{CHO}) ;{ }^{13} \mathrm{C}-\mathrm{NMR}(125 \mathrm{MHz}$, $\left.\mathrm{CDCl}_{3}\right) \delta_{\mathrm{C}}: 129.8(\mathrm{C}-1), 108.9(\mathrm{C}-2), 146.8(\mathrm{C}-3), 146.2(\mathrm{C}-4), 114.3(\mathrm{C}-5), 127.7(\mathrm{C}-6), 191.0(\mathrm{C}=\mathrm{O})$.

\section{Results and Discussion}

From the $\mathrm{CDCl}_{3}$ of $A$. ordosica, six compounds were obtained using chromatographic methods (CC and TLC). On the basis of ${ }^{1} \mathrm{H},{ }^{13} \mathrm{C}$ NMR, COSY, HSQC, HMBC and HR-ESI-MS spectra, and modified Mosher's method as well as by comparison with previous reports [9-13], compounds 1 was identified as a coumarin with new skeleton while the remaining eight compounds were found to be the known compounds, 6,7-dimethoxycoumarin (2), 6,7,8-trimethoxycoumarin (3), 6-hydroxy-7methoxycoumarin (4), 4-hydroxylacetophenone (5), 4-hydroxy-5-methoxylacetophenone (6), 4hydroxybenzaldehyde (7), 4-hydroxy-5-methoxybenzaldehyde (8) and 4,5-dihydroxybenzaldehyde (9) (Figure 1).

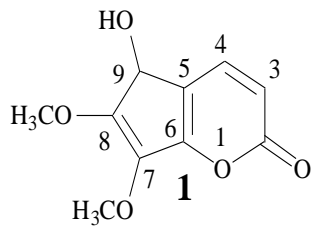<smiles>COc1cc2ccc(=O)oc2cc1OC</smiles><smiles>COc1cc2ccc(=O)oc2c(OC)c1OC</smiles><smiles>COc1cc2oc(=O)ccc2cc1O</smiles><smiles>CC(=O)c1ccc(O)cc1</smiles><smiles>COc1cc(C(C)=O)ccc1O</smiles><smiles>Oc1ccc(C=Cc2ccccc2)cc1</smiles><smiles>COc1cc(C=O)ccc1O</smiles><smiles>O=Cc1ccc(O)c(O)c1</smiles>

Figure 1. Structures of compounds 1-9

Compound 1 was obtained as a white needle, mp 137-139 ${ }^{\circ} \mathrm{C}$; IR $(\mathrm{KBr}) v_{\max }\left(\mathrm{cm}^{-1}\right): 3312,1683$, 1643 and $1320 \mathrm{~cm}^{-1}$. The molecular formula was determined to be $\mathrm{C}_{10} \mathrm{H}_{10} \mathrm{O}_{5}$ by HR-ESI-MS exhibiting a pseudomolecular ion peak at $\mathrm{m} / z 209.0467[\mathrm{M}-\mathrm{H}]^{-}$(calcd for $\mathrm{C}_{10} \mathrm{H}_{9} \mathrm{O}_{5}, 209.0450$ ). In the ${ }^{1} \mathrm{H}$ NMR spectrum (Table 1), two characteristic resonances for $\mathrm{H}-3$ and $\mathrm{H}-4$ of a coumarin at $\delta_{\mathrm{H}} 6.03(1 \mathrm{H}, \mathrm{d}, J=$ $10.0 \mathrm{~Hz}, \mathrm{H}-3)$ and $8.06(1 \mathrm{H}, \mathrm{d}, J=10.0 \mathrm{~Hz}, \mathrm{H}-4)$. In addition, the signals at $\delta_{\mathrm{H}} 3.64(3 \mathrm{H}, \mathrm{s})$ and 3.82 $(3 \mathrm{H}, \mathrm{s})$ indicated the presence of two methoxy groups. The remaining signal at $\delta_{\mathrm{H}} 6.39(1 \mathrm{H}, \mathrm{s})$ was assigned to $\mathrm{H}-9$ compared with the data of $\mathrm{H}-25\left(\delta_{\mathrm{H}} 6.21\right)$ in fasciospongides $\mathbf{A}$ [14], which was confirmed by the HMBC correlations (Figure 2$)$ from $\delta_{\mathrm{H}} 6.39(1 \mathrm{H}, \mathrm{s}, \mathrm{H}-9)$ to C-5 $\left(\delta_{\mathrm{C}} 104.8\right), \mathrm{C}-6\left(\delta_{\mathrm{C}} 152.3\right)$ and C$7\left(\delta_{\mathrm{C}} 133.5\right)$.

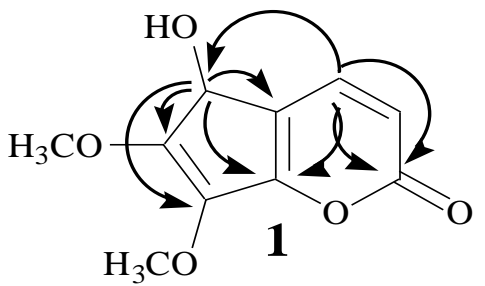

Figure 2. Selected HMBC correlations for 1 
Table 1. ${ }^{1} \mathrm{H}(500 \mathrm{MHz})$ and ${ }^{13} \mathrm{C}-\mathrm{NMR}(125 \mathrm{MHz})$ data of compound $\mathbf{1}$ in DMSO- $d_{6}$

\begin{tabular}{lll}
\hline Position & $\delta_{\mathrm{H}}(\mathrm{ppm}), J(\mathrm{~Hz})$ & $\delta_{\mathrm{c}}(\mathrm{ppm})$ \\
\hline 2 & - & 161.5 \\
3 & $6.03 \mathrm{~d}(10.0)$ & 109.1 \\
4 & $8.09 \mathrm{~d}(10.0)$ & 141.1 \\
5 & - & 104.8 \\
6 & - & 152.3 \\
7 & - & 133.5 \\
8 & - & 157.0 \\
9 & $6.39 \mathrm{~s}$ & 89.7 \\
$7-\mathrm{OCH}_{3}$ & $3.64 \mathrm{~s}$ & 60.6 \\
$8-\mathrm{OCH}_{3}$ & $3.82 \mathrm{~s}$ & 56.5 \\
\hline
\end{tabular}

The ${ }^{13} \mathrm{C}$ NMR spectrum of 1 showed 10 carbon signals, of which 8 were assigned to the coumarin skeleton part and 2 to the two methoxy groups. In coumarin skeleton part, there were only 8 carbon signals $\left(\delta_{\mathrm{C}} 161.5,109.1,141.8,104.8,152.3,133.5,157.0,89.7\right)$, which was different from the 9 carbon signals of an usual coumarin skeleton $[9,10]$. The benzene in an usual coumarin skeleton was substituted by the 1,3-diene cyclopentane in the new coumarin skeleton, in which the HMBC correlations from $\mathrm{H}$ 4 to C-2 $(\delta \mathrm{c} 161.5), \mathrm{C}-6(\delta \mathrm{c} 152.3)$ and C-9 $(\delta \mathrm{c} 89.7)$, and H-3 to C-2 $(\delta \mathrm{c} 161.5)$ and C-5 $(\delta \mathrm{c} 104.8), \mathrm{H}-$ 9 to $\mathrm{C}-5\left(\delta_{\mathrm{C}} 104.8\right), \mathrm{C}-6\left(\delta_{\mathrm{C}} 152.3\right)$ and C-7 $\left(\delta_{\mathrm{C}} 133.5\right)$ were show. In addition, the HMBC correlations $\delta_{\mathrm{H}}$ $3.64\left(-\mathrm{OCH}_{3}\right)$ to $\delta \mathrm{c} 133.5(\mathrm{C}-7)$ and $3.82\left(-\mathrm{OCH}_{3}\right)$ to $\delta \mathrm{c} 157.0(\mathrm{C}-8)$ revealed that the two methoxy groups were linked to the $\mathrm{C}-7$ and $\mathrm{C}-8$, respectively. The modified Mosher's method was used to produce $(R)$ - and $(S)$-MTPA esters $(\mathbf{1 a}, \mathbf{1 b})$, and signals corresponding to $\mathrm{H}-3, \mathrm{H}-4$ and $8-\mathrm{OCH}_{3}$ were relatively deshielded in $\mathbf{1 a}$ compared to $\mathbf{1 b}$, indicating that the absolute configuration of C-9 is $S$ (Figure 3 ). Thus, the structure of compound $\mathbf{1}$ was elucidated and named as arteordocoumarin A.

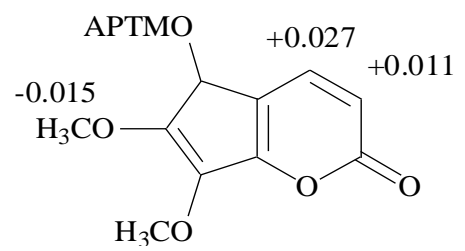

Figure 3. Results with the modified Mosher's method $\left(\Delta=\delta_{S}-\delta_{R}\right)$ for compound 1

\section{Acknowledgments}

This work was supported by the Science and Technology Major Project of Inner Mongolia Mongolian medicine in China (No. GCY20150712) and Graduate Research Innovation Projects of Inner Mongolia (No. B20161013601).

\section{Supporting Information}

Supporting information accompanies this paper on http://www.acgpubs.org/journal/records-ofnatural-products

\section{ORCID}

Wugedunqiqige Bao: 0000-0003-0744-8361

Qinghu Wang: 0000-0002-6272-6398

Junsheng Hao: $\underline{0000-0002-7733-133 \mathrm{X}}$ 


\section{References}

[1] M.J. Abad, L.M. Bedoya, L. Apaza and P. Bermejo (2012). The Artemisia L. genus: A review of bioactive essential oils, Molecules 17, 2542-2566.

[2] H. Zhang, D. Zhou, Y. Luo, J. Wang and S. Zong (2013). Identification of volatile compounds emitted by Artemisia ordosica (Artemisia, Asteraceae) and changes due to mechanical damage and weevil infestation, Z. Naturforsch. C. 68, 313-317.

[3] Z. Zhang, S.S. Guo, W.J. Zhang, Z.F. Geng, J.Y. Liang, S.S. Du, C.F. Wang and Z.W. Deng (2017). Essential oil and polyacetylenes from Artemisia ordosica and theirbioactivities against Tribolium castaneum Herbst (Coleoptera:Tenebrionidae), Ind. Crop. Prod. 100, 132-137.

[4] D.B. Zhao, Y.F. Jiang, C.J. Xi, X.H. Liu and H.Q. Wang (2005). Isolation and identification of capillarin from Artemisia ordosica Krasch, Chin. J. Struct. Chem. 24, 637-640

[5] R.X. Tan, W.F. Zheng and H.Q. Tang (1998). Biologically active substances from the genus Artemisia, Planta. Med. 64, 295-302

[6] Y. Zhong, X.S. Feng and Y.R. Liu (2016). Chemical constituents from Artemisia ordosica Krasch and their bioactivities, Chem. Bioeng. 33, 36-38.

[7] D.B. Zhao, Y.X. Yang, W. Zhang, X.H. Liu and H.Q. Wang (2005). Studies on flavonoid constituents from herbs of Artemisia ordosica II, Chin. J. Chin. Mater. Med. 31, 1959-1961.

[8] W. Zhang, D.B. Zhao, M.J. Li, X.H. Liu and H.Q. Wang (2006). Studies on flavonoid constituents from herbs of Artemisia ordosica II, Chin. J. Chin. Mater. Med. 30, 1430-1432.

[9] J.Y. Wu, Y.B. Wu, J. Yi, T. Wang, Z.H. Huang, J.G. Wu and J.Z. Wu (2013). Chemical constituents in leaves of Magnolia officinalis var. biloba, Chin. Trad. Herb. Drag. 44, 2965-2968.

[10] C. Kofinas, I. Chinou, A. Loukis, C. Harvala and C. Roussakis (1998). Cytotoxic coumarins from the aerial parts of Tordylium apulum and their effects on a non-small-cell bronchial carcinoma line, Planta. Med. 64,171-179.

[11] L.L. Song, X.W. Li and F.F. Yan (2010). study on the chemical constituents of Daphne genkwa, Chin. Trad. Herb. Drag. 41, 536-539.

[12] Y.W. Wang, Z.F. Li, M.Z. Zhen, Y.L. Feng, Q. Wang, X. Li and S.L. Yang (2013). Chemical constituents of Gastrodia elata, Chin. Trad. Herb. Drag. 44, 2794-2796.

[13] X.H. Duan, Z.L. Li, D.S. Yang, F.L. Zhang, Q. Lin and R. Dai (2013). Study on the chemical constituents of Gastrodia elata, J. Chin. Med. Mat. 36, 1608-1611.

[14] A. Montagnac, M. Pas and C. Debitus (1994). Fasciospongides A, B and C, new manoalide derivatives from the sponge fasciospongia SP, J. Nat. Prod. 57, 186-190.

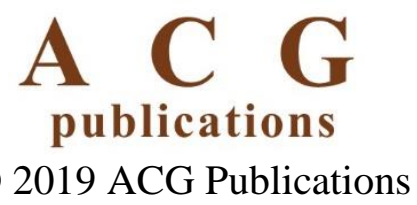

